\title{
OPTIMAL EVACUATION PLAN DESIGN WITH IP-SOLVER
}

This paper deals with two different computer-supported approaches to an evacuation plan design, which should assign the available vehicles to endangered dwelling places so that the total time of evacuation is minimal. It is assumed that some safe place is pre-assigned to each endangered dwelling place and there are determined locations of homogenous fleets of available vehicles, which can be used for transport of the endangered population from their dwelling places to the pre-assigned places. In this paper, we suggest and compare two approaches to the evacuation plan design. The first approach assigns all vehicles of a fleet to one evacuated dwelling place and the second one enables to assign individually any part of a fleet to the dwelling places.

\section{Introduction}

Let us consider an emergency situation when population of a given set of towns and villages is endangered by some threat. The casualties can be avoided to some extent by evacuation of the endangered population to some safe places, which have been predestinated for each evacuated place in advance. To perform the evacuation, some available vehicles are disposable at several places located in the neighborhood of the endangered dwelling places. It is necessary to determine a route for each used vehicle so that the population is evacuated from its original places to the predetermined refuges. The evacuation should be performed so that the time of evacuation is as short as possible. The end of evacuation is given by the time when the last inhabitant reaches his/her predestinated shelter.

If any vehicle is used to provide an evacuated community with this service, the route of vehicle may take a prescribed form. The route starts at the original vehicle location, continues to the served village or town, picks up a portion of the evacuated inhabitants and takes them to the predetermined refuge [4]. If necessary, the vehicle may return back to the evacuated place and save another portion of its population by taking them to the refuge. This cycle can be repeated several times.

Even under this simplifying assumption about a form of route, time optimal assignment of the vehicles to the evacuated places represents a hard combinatorial problem, whose solution must be usually found in a short time of several minutes. In this paper, we present different approaches to this problem. Each of these approaches enables to employ a commercial IP-solver, to obtain a final concrete set of decisions on the vehicle assignment. All these approaches consist of a linear programming model formulation and solving process performed by commercial software with usage of its particular characteristics. The presented approaches differ in models and following quality of obtained solutions. These properties were studied by numerical experiments and their results are presented in the concluding part of this paper.

To formulate the following mathematical models for the individual approaches, we shall use a common denotation, where symbol $I$ denotes the set of all considered homogenous fleets of vehicles. Each homogenous fleet $i \in I$ is characterized by a number $N_{i}$ of vehicles and by vehicle capacity $K_{i}$. We shall assume that the fleet $i$ is located at a node $u(i)$ of a road network covering the serviced area. The endangered dwelling places form a set $J$ and each dwelling place $j \in J$ is described by a number $b_{j}$ of its population and by a road network node $v(j)$, where the village or town $j$ is located. We assumed for the purpose of evacuation that a destination place $w(j)$ is assigned to each dwelling place $j \in J$. Furthermore, let $t_{i j}$ denote the time, which is necessary for a vehicle from the fleet $i$ to traverse the distance between the nodes $u(i)$ and $v(j)$. In addition, let $s_{j}$ denote the time necessary for traversing the distance between the nodes $v(j)$ and $w(i)$. Using these denotations, we can introduce two approaches to the evacuation plan design problem. The first approach assumes that each considered homogenous fleet is indivisible, i.e. all the vehicles of one fleet perform simultaneously the same activities like a convoy or vehicle train. The second approach is based on the assumption that each fleet can be split into arbitrary integer parts and only vehicles of one part have to act as a convoy.

The first approach leads to a simpler but larger model, whereas the second one pays for its smaller size by non-linearity of the associated model. Advantages and disadvantages of the both approaches are studied in the next two sections and some results of numerical experiments are presented in the concluding part of this paper to demonstrate efficiency of the both approaches, in

\footnotetext{
* Jaroslav Janacek ${ }^{1}$, Michal Sibila ${ }^{2}$

${ }^{1}$ Department of Transportation Networks, Faculty of Management and Informatics, University of Zilina, Slovakia, E-mail: jaroslav.janacek@fri.uniza.sk,

${ }^{2}$ Department of Technical Sciences and Informatics, Faculty of Special Engineering, University of Zilina, Slovakia
} 
the case when a commercial software tool is used for obtaining final decisions on the evacuation plan.

\section{Evacuation problem with indivisible fleets}

\subsection{Problem formulation and model building}

Let us consider that a set $J$ represents evacuated dwelling places where each element $j \in J$ is characterized by a location $v(j)$, a number of population $b_{j}$ and a location $w(j)$, to which all population must be transported. For this purpose a set $I$ of indivisible fleets is disposable where each fleet $i \in I$ is characterized by a location $u(i)$, a number $N_{i}$ of identical vehicles and a vehicle capacity $K_{i}$. Let $t_{i j}$ and $s_{j}$ be travelling times between the locations $u(i)$ and $v(j)$ and between $v(j)$ and $w(j)$ respectively. The objective is to determine a route of each indivisible fleet so that all population is evacuated and the longest fleet route is minimal [3].

Coming out of the assumption on the possible form of a fleet route, we introduce the variable $z_{i j} \in\{0,1\}$ defined for each pair of the fleet $i$ and dwelling place $j$. This variable takes the value of 1 if and only if the fleet $i$ is assigned to the dwelling place $j$.

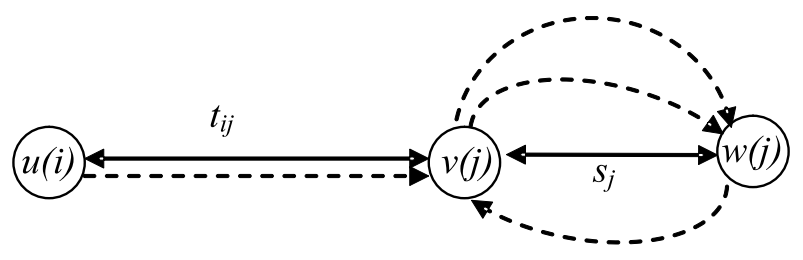

Fig. 1 A route with two visits at $v(j)$

Taking into account that the fleet $i$ can visit an evacuated place several times, we denote the number of journeys of fleet $i$ from $v(j)$ to $w(j)$ by variable $x_{i j} \in Z^{+}$. Now, when the fleet $i$ is assigned to the dwelling place $j$, its travelling time (see Fig. 1) is equal to:

$$
t_{i j}+s_{j}+2 s_{j}\left(x_{i j}-1\right)
$$

The last introduced variable is $T \geq 0$, which denotes an upper bound of all route times.

We can take into account that a maximal sensible time $T^{\max }$ of the evacuation can be given. In this case the maximal possible number of visits of fleet $i$ at the place $j$ can be evaluated to comply with this limit. The inequality (2) must hold for the $x_{i j}$.

$$
t_{i j}+s_{j}+2 s_{j}\left(x_{i j}-1\right) \leq T^{\max }
$$

The inequality (2)can be rewritten as:

$$
x_{i j} \leq\left\lfloor\frac{T^{\max }-t_{i j}+s_{j}}{2 s_{j}}\right\rfloor
$$

Denote $P_{i j}\left(T^{\max }\right)=\left\lfloor\frac{T^{\max }-t_{i j}+s_{j}}{2 s_{j}}\right\rfloor$.

If $P_{i j}\left(T^{\max }\right) \leq 0$, then the fleet $i$ is not able to service the place $j$. To minimize the size of a built model, we introduce a set $J(i)$ of all the places from $J$, for which the inequality $P_{i j}\left(T^{\max }\right)>0$ holds. Similarly, we define a set $I(i)$ of all the fleets from $I$, for which $P_{i j}\left(T^{\max }\right)>0$ holds.

The linear model of an evacuation plan design can be completed now as follows:

Minimize T

$$
\begin{aligned}
\text { Subject to } & \left(t_{i j}-s_{j}\right) z_{i j}+2 s_{j} x_{i j} \leq T \text { for } i \in I, j \in J(i) \\
& \sum_{j \in J(i)} z_{i j} \leq 1 \text { for } i \in I \\
& x_{i j} \leq P_{i j}\left(T^{\max }\right) z_{i j} \text { for } i \in I, j \in J(i) \\
& \sum_{i \in((j)} N_{i} K_{i} x_{i j} \geq b_{j} \text { for } j \in J \\
& z_{i j} \in\{0,1\} \text { for } i \in I, j \in J(i) \\
& z_{i j} \in Z^{+} \text {for } i \in I, j \in J(i) \\
& T \geq 0
\end{aligned}
$$

The constraints (5) assure that the travelling time of each fleet is less or equal to the upper bound $T$. The constraints (6) enable for the fleet $i$ to be assigned at most to one evacuated dwelling place. Constraints (7) cause that if the variable $z_{i j}$ is equal to zero, then the variable $x_{i j}$ is also zero, which means that if the fleet $i$ is not assigned to the place $j$, then the fleet cannot visit this place at all. The constraints (8) ensure that each dwelling place $j$ is provided with a sufficient capacity, which enables to evacuate all the population of the size $b_{j}$.

\subsection{Numerical experiments}

To verify the suggested use of the IP-solver, we formulated ten different instances of the problem. One of the instances denoted as "Hradza" comes out of the possible emergency situation, which can occur if the dam Liptovska Mara breaks. Then, under given assumptions, 26 communities would have to be evacuated to 26 predestined places. For this evacuation, 411 vehicles of different capacities located at three bigger towns of the area are available. The other instances were formulated in nine areas of the Slovak Republic in a similar way. These instances are denoted by names of the biggest towns of the areas. The numbers and capacities of available vehicles were generated similarly to the first instance for the vehicles to be able to satisfy the demand on evacuation. These benchmarks were used to verify the suggested method, which consists in a particular model building and employing the general IPsolver for obtaining of a good solution of the problem. To be able to perform the computation in a given time, we used the general 
optimisation software environment XPRESS-IVE for our study [5], [6]. This software system includes the branch-and-cut method and it also enables exploitation of the premature stopping rules. The software is equipped with the programming language Mosel, which can be used for both the input of model and writing of input and output procedures. Furthermore, the language has its own tools for the stopping rules adjustment.

The main disadvantage is born-in to the branch-and-bound method, which is the basic solving method of any IP-solver. This is the possibility that the list of determined but unfathomed solution subsets may grow exponentially instead of being reduced to an empty set. When a special IP-solver is designed, all specific properties of the problem can be used to improve quality of the upper and lower bounds, whereas only a general problem relaxation can be employed in the case of this general solver. We used the possibility of the solver, which enables a premature termination of the searching process whenever a fixed time limit is exceeded. The experiments were performed on a personal computer equipped with Intel Core 26700 with parameters: $2.66 \mathrm{GHz}$ and $3 \mathrm{~GB}$ RAM. The first series of experiments corresponds with the case, when each vehicle is considered as one individual fleet. The best results obtained in the computational time of 20 minutes are presented in Table 1, where "No. of F" denotes the considered number of fleets, "No. of EP" denotes the number of evacuated dwelling places, Rows denotes the number of structural constraints of the model and Columns denotes the number of used variables including the auxiliary ones, which are automatically introduced by the solver. In the row denoted as "Tmax", there are reported the predetermined values of $T^{\max }$ in minutes. The symbol "Tbest" denotes the row, where the bestfound time of evacuation is plotted.

As the preliminary experiments showed that decreasing the parameter $T^{\max }$ to diminish the numbers of constraints and vari-

Results of numerical experiments for indivisible fleets of one vehicle

Table 1

\begin{tabular}{|l|c|c|c|c|c|c|c|c|c|c|}
\hline \multicolumn{1}{|c|}{ Instances: } & Bratislava & $\begin{array}{c}\text { Dubnica } \\
\text { nad } \\
\text { Vahom }\end{array}$ & Hradza & Kosice & $\begin{array}{c}\text { Liptovsky } \\
\text { Mikulas }\end{array}$ & Leopoldov & Michalovce & Nitra & $\begin{array}{c}\text { Nove } \\
\text { Zamky }\end{array}$ & Puchov \\
\hline No. of F. & 635 & 183 & 411 & 483 & 107 & 281 & 105 & 211 & 107 & 435 \\
\hline No. of EP. & 25 & 25 & 26 & 25 & 25 & 25 & 25 & 25 & 25 & 25 \\
\hline Rows & 16958 & 4966 & 11485 & 11751 & 2914 & 7331 & 2860 & 5722 & 2914 & 10853 \\
\hline Columns & 31539 & 9151 & 21324 & 22836 & 5351 & 13770 & 5251 & 10551 & 5351 & 20834 \\
\hline Tmax [min] & 162 & 938 & 202 & 184 & 668 & 140 & 1420 & 423 & 1497 & 278 \\
\hline Tbest [min] & $\mathbf{8 1}$ & $\mathbf{4 3 4}$ & $\mathbf{9 3}$ & $\mathbf{9 2}$ & $\mathbf{2 9 4}$ & $\mathbf{1 0 1}$ & $\mathbf{5 8 6}$ & $\mathbf{1 8 2}$ & $\mathbf{7 0 4}$ & $\mathbf{1 3 9}$ \\
\hline
\end{tabular}

Results of numerical experiments for indivisible fleets of two vehicles

Table 2

\begin{tabular}{|l|c|c|c|c|c|c|c|c|c|c|}
\hline \multicolumn{1}{|c|}{ Instances: } & Bratislava & $\begin{array}{c}\text { Dubnica } \\
\text { nad } \\
\text { Vahom }\end{array}$ & Hradza & Kosice & $\begin{array}{c}\text { Liptovsky } \\
\text { Mikulas }\end{array}$ & Leopoldov & Michalovce & Nitra & $\begin{array}{c}\text { Nove } \\
\text { Zamky }\end{array}$ & Puchov \\
\hline No. of F. & 323 & 92 & 209 & 243 & 56 & 144 & 55 & 107 & 54 & 219 \\
\hline No. of EP. & 25 & 25 & 26 & 25 & 25 & 25 & 25 & 25 & 25 & 25 \\
\hline Rows & 8638 & 2509 & 5789 & 5852 & 1494 & 3769 & 1504 & 2914 & 1483 & 5456 \\
\hline Collums & 16043 & 4601 & 10780 & 11417 & 2758 & 7057 & 2745 & 5351 & 2701 & 10469 \\
\hline Tmax [min] & 162 & 938 & 202 & 184 & 668 & 140 & 1420 & 423 & 1497 & 278 \\
\hline Tbest [min] & $\mathbf{8 1}$ & $\mathbf{4 9 5}$ & $\mathbf{9 3}$ & $\mathbf{9 2}$ & $\mathbf{3 2 8}$ & $\mathbf{1 0 8}$ & $\mathbf{5 8 4}$ & $\mathbf{1 9 4}$ & $\mathbf{7 5 0}$ & $\mathbf{1 3 9}$ \\
\hline
\end{tabular}

Results of numerical experiments for indivisible fleets of four vehicles

Table 3

\begin{tabular}{|l|c|c|c|c|c|c|c|c|c|c|}
\hline \multicolumn{1}{|c|}{ Instances: } & Bratislava & $\begin{array}{c}\text { Dubnica } \\
\text { nad } \\
\text { Vahom }\end{array}$ & Hradza & Kosice & $\begin{array}{c}\text { Liptovsky } \\
\text { Mikulas }\end{array}$ & Leopoldov & Michalovce & Nitra & $\begin{array}{c}\text { Nove } \\
\text { Zamky }\end{array}$ & Puchov \\
\hline No. of F. & 171 & 46 & 113 & 126 & 35 & 78 & 33 & 57 & 31 & 115 \\
\hline No. of EP. & 25 & 25 & 26 & 25 & 25 & 25 & 25 & 25 & 25 & 25 \\
\hline Rows & 4576 & 1267 & 2864 & 3024 & 826 & 2053 & 898 & 1564 & 854 & 2745 \\
\hline Columns & 8485 & 2301 & 5551 & 5898 & 1538 & 3823 & 1633 & 2851 & 1543 & 5366 \\
\hline Tmax [min] & 162 & 938 & 202 & 184 & 668 & 140 & 1420 & 423 & 1497 & 278 \\
\hline Tbest [min] & $\mathbf{8 1}$ & $\mathbf{5 1 0}$ & $\mathbf{9 3}$ & $\mathbf{9 2}$ & $\mathbf{3 8 1}$ & $\mathbf{1 0 5}$ & $\mathbf{7 9 6}$ & $\mathbf{1 9 8}$ & $\mathbf{1 0 9 1}$ & $\mathbf{1 3 9}$ \\
\hline
\end{tabular}


Results of numerical experiments for indivisible fleets of eight vehicles

Table 4

\begin{tabular}{|l|c|c|c|c|c|c|c|c|c|c|}
\hline \multicolumn{1}{|c|}{ Instances: } & Bratislava & $\begin{array}{c}\text { Dubnica } \\
\text { nad } \\
\text { Vahom }\end{array}$ & Hradza & Kosice & $\begin{array}{c}\text { Liptovsky } \\
\text { Mikulas }\end{array}$ & Leopoldov & Michalovce & Nitra & $\begin{array}{c}\text { Nove } \\
\text { Zamky }\end{array}$ & Puchov \\
\hline No. of F. & 98 & 28 & 71 & 71 & 23 & 48 & 24 & 33 & 22 & 65 \\
\hline No. of EP. & 25 & 25 & 26 & 25 & 25 & 25 & 25 & 25 & 25 & 25 \\
\hline Rows & 2555 & & 1652 & 1687 & & 1273 & & 888 & & 1376 \\
\hline Columns & 4785 & & 3331 & 3296 & & 2353 & & 1623 & & 2847 \\
\hline Tmax [min] & 162 & 938 & 202 & 184 & 668 & 140 & 1420 & 423 & 1497 & 278 \\
\hline Tbest [min] & $\mathbf{8 1}$ & $*$ & $\mathbf{9 3}$ & $\mathbf{9 2}$ & $*$ & $\mathbf{1 1 9}$ & $*$ & $\mathbf{2 7 2}$ & $*$ & $\mathbf{1 3 9}$ \\
\hline
\end{tabular}

ables of the model had no effect on obtaining a better solution in the given time limit, we decided to explore another way of a model size diminishing. We formed models with a smaller number of fleets so that we grouped the vehicles by two, four and eight if possible to fleets of several vehicles with the same capacity. The derived problems were solved and the results are presented in tables 2, 3 and 4 respectively.

It can be observed that the process of forming the bigger fleets ended at eight-vehicle fleets for some instances, when the solving process fails in finding a solution due to infeasibility. These cases are denoted by asterisk in the row "Tbest". Furthermore, the found resulting evacuation times turned worse for instances, where a feasible solution was found. This result evoked an idea to formulate the evacuation problem for divisible fleets, which is the topic of the next section.

\section{Evacuation problem with divisible fleets}

\subsection{Problem formulation and model building}

Similarly to the previous section, we consider that the set $J$ represents evacuated dwelling places, where each element $j \in J$ is characterized by a location $v(j)$, a number of population $b_{j}$ and a location $w(j)$, to which all population must be transported. For this purpose the set $I$ of divisible fleets is disposable, where each fleet $i \in I$ is characterized by a location $u(i)$, a number $N_{i}$ of identical vehicles and a capacity $K_{i}$ of an individual vehicle. Let $t_{i j}$ and $s_{j}$ be travelling times between the locations $u(i)$ and $v(j)$ and between $v(j)$ and $w(j)$ respectively. The objective is to determine a route of each part of divisible fleet so that all the population be evacuated and the longest route be minimal.

Coming out of the assumption on the possible form of a route, which stays the same as depicted in Fig. 1, we introduce the variables $z_{i j} \in\{0,1\}$ and $x_{i j} \in Z^{+}$defined for each pair of fleet $i$ and dwelling place $\mathrm{j}$ as before. In addition to these variables, we introduce the variables $q_{i j} \in Z^{+}$, which denote a number of vehicles of the fleet $i$ assigned to the dwelling place $j$.

Taking into account expressions (1) and (3) a model of the evacuation plan design with divisible fleets can be written as follows:
Minimize T

$$
\begin{array}{ll}
\text { Subject to } & \left(t_{i j}-s_{j}\right) z_{i j}+2 s_{j} x_{i j} \leq T \text { for } i \in I, j \in J(i) \\
& x_{i j} \leq P_{i j}\left(T^{\mathrm{max}}\right) z_{i j} \text { for } i \in I, j \in J(i) \\
& q_{i j} \leq N_{i} z_{i j} \text { for } i \in I, j \in J(i) \\
& \sum_{j \in J(i)} q_{i j} \leq N_{i} \text { for } i \in I, \\
& \sum_{i \in I(j)} K_{i} q_{i j} x_{i j} \geq b_{j} \text { for } j \in J \\
& z_{i j} \in\{0,1\} \text { for } i \in I, j \in J(i) \\
& x_{i j} \in Z^{+} \text {for } i \in I, j \in J(i) \\
& \mathrm{T} \geq 0 \\
& q_{i j} \in Z^{+} \text {for } i \in I, j \in J(i)
\end{array}
$$

The constraints (13) assure that the travelling time of each part of the fleet is less or equal to the upper bound $T$. The constraints (14) represent binding constraints between the variables $x_{i j}$ and $z_{i j}$. These constraints cause that if the variable $z_{i j}$ is equal to zero, then the variable $x_{i j}$ is also zero, which means that if the fleet $i$ is not assigned to the place $j$, then the fleet cannot visit this place at all. The constraints (15) represent binding constraints between the variables $q_{i j}$ and $z_{i j}$. These constraints cause that if the variable $z_{i j}$ is equal to zero, then the variable $q_{i j}$ is also zero, which means that if the fleet $i$ is not assigned to the place $j$, then no vehicle of the fleet $i$ can visit this place. The constraints (16) assure that the total number of designed vehicles of the fleet $i$ does not exceed the number $N_{i}$.

The constraints (17) ensure that each dwelling place $j$ is provided with a sufficient capacity, which enables to evacuate all the population of size $b_{j}$. Unfortunately, these constraints are non-linear and thus this model cannot be input to the IP-solver. To be able to solve this much smaller problem, it must be rearranged to a linear form. An approach to the model linearization is shown in the next section. 


\subsection{Problem reformulation to a linear model}

To rewrite the model into a linear form, we make use of the fact that the variable $x_{i j}$ may take only one of several few values from the range of $0,1, \ldots, P_{i j}$. We introduce auxiliary variables $m_{i j}$, which serve as a lower bound of the number of visits at the dwelling place $j$, which are performed by the vehicles of fleet $i$. Then the following constrains must hold.

$$
q_{i j} x_{i j} \geq m_{i j} \quad \text { for } i \in I, j \in J(i)
$$

Now the series (17) of constraints can be replaced by the constraints (23).

$$
\sum_{i \in I(j)} K_{i} m_{i j} \geq b_{j} \quad \text { for } j \in J
$$

Now the constraint $q_{i j} x_{i j} \geq m_{i j}$ can be replaced by the following system of logical constraints:

$$
\begin{aligned}
& \text { If } x_{i j}=0 \text { then } 0 \geq m_{i j} . \\
& \text { If } x_{i j}=1 \text { then } q_{i j} \geq m_{i j} . \\
& \text { If } x_{i j}=2 \text { then } 2 q_{i j} \geq m_{i j} . \\
& \ldots \\
& \text { If } x_{i j}=k \text { then } k q_{i j} \geq m_{i j} . \\
& \ldots \\
& \text { If } x_{i j}=P_{i j}-1 \text { then }\left(P_{i j}-1\right) q_{i j} \geq m_{i j} .
\end{aligned}
$$

It holds here that if some of the constraints is fulfilled for $k=p$, then they are fulfilled for each $k>p$ and, vice versa, if a constraint is not fulfilled for $k=p$, then they cannot be fulfilled for any $k<p$.

Now we introduce variables $y_{i j}^{p} \in\{0,1\}$ for $i \in I$ and $j \in J(i)$ and $p=0,1, \ldots, P_{i j}-1$ and the system of logical constraints can be replaced by the series (24) of constraints.

$$
\begin{aligned}
& N_{i} P_{i j} y_{i j}^{p}+p q_{i j} \geq m_{i j} \\
& \text { for } i \in I, j \in J(i), p=0,1, \ldots, P_{i j}-1
\end{aligned}
$$

If the system (24) holds for given values of variables $q_{i j}$ and $k$ is the minimal value of subscript $p$ for which $y_{i j}^{k}=0$, then the system must also hold for such a setting of variables $y_{i j}^{p}$, where $y_{i j}^{p}=1$ for $p=0,1, \ldots, k-1$ and $y_{i j}^{p}=0$ for $p=k, \ldots, P_{i j}-1$, i.e. the setting of variables $y_{i j}^{p}$ fulfils the equation (25).

$$
\sum_{p=0}^{P_{i j}-1} y_{i j}^{p}=k \quad \text { for } i \in I, j \in J(i)
$$

Then the following linear model describes the evacuation plan design problem with divisible fleets:

\section{Minimize T}

$$
\text { Subject to }\left(t_{i j}-s_{j}\right) z_{i j}+2 s_{j} \sum_{p=0}^{P_{j}\left(T^{\mathrm{max}}\right)-1} y_{i j}^{p} \leq T
$$$$
\text { for } i \in I, j \in J(i)
$$

$$
\begin{aligned}
& \sum_{p=0}^{P_{i j}\left(T^{\operatorname{mx}}\right)-1} y_{i j}^{p} \leq P_{i j}\left(T^{\max }\right) z_{i j} \text { for } i \in I, j \in J(i) \\
& q_{i j} \leq N_{i} z_{i j} \text { for } i \in I, j \in J(i) \\
& \sum_{j \in J(i)} q_{i j} \leq N_{i} \text { for } i \in I \\
& \left.\sum_{i \in((j)} K_{i} m_{i j} \geq b_{j} \text { forj } \in\right) J \\
& N_{i} P_{i j}\left(T^{\max }\right) \geq m_{i j} \text { for } i \in I, j \in J(i) \\
& N_{i} P_{i j} y_{i j}^{p}+p q_{i j} \geq m_{i j} \\
& \text { for } i \in I, j \in J(i), p=0, \ldots, P_{i j}\left(T^{\max }\right)-1 \\
& z_{i j} \in\{0,1\} \quad \text { for } i \in I, j \in J(i) \\
& T \geq 0 \\
& m_{i j} \geq 0 \quad \text { for } i \in I, j \in J(i) \\
& y_{i j}^{p} \in\{0,1\} \\
& \text { for } i \in I, j \in J(i), p=0, \ldots, P_{i j}\left(T^{\max }\right)-1
\end{aligned}
$$

The constraints (27) have the same meaning as the constraints (13) in the non-linear model (12) - (21), i.e. they assure that the travelling time of each part of the fleet is less than the upper bound $T$. These constraints were derived from the constraints (13) by substitution of the left-hand-side of the equality (25) for $x_{i j}$. The constraints (28) are the binding constraints, which assure relations between the variables $z_{i j}$ and the sum of $y_{i j}^{p}$, which corresponds with the number $x_{i j}$ of visits of the part of the fleet $i$ at the community $j$. The constraints (29) were equivalent to the former constraints (15), which assure that if no part of the fleet $i$ is designated to the place $j\left(z_{i j}=0\right)$, then the number $q_{i j}$ of vehicles is equal to zero. The constraints (30) assure that the total number of designed vehicles of the fleet $i$ does not exceed the number $N_{i}$.

As $m_{i j}$ represents a lower bound of the product $x_{i j} q_{i j}$, which is the number of visits of vehicles from the fleet $i$ at the place $j$, then the constraints (31) ensure that population of the place $j$ can be evacuated. The constraints (32) ensure that the estimation $m_{i j}$ does not exceed the upper bound $N_{i} P_{i j}\left(T^{\max }\right)$ of visits and the constraints (33) ensure that the sum of $y_{i j}^{p}$ over $p$ corresponds with the number of necessary visits of a group of $q_{i j}$ vehicles of the fleet $i$ at $j$.

\subsection{Numerical experiments}

To find characteristics of the second approach involving the linear model of evacuation by divisible fleets, we solved the same instances, which are described in section 2.3. We used the same setting of $T^{\max }$ and we focused on the evacuation times, which were obtained in the computational time of 20 minutes. The results associated with these experiments are reported in Table 5. 
Results of numerical experiments for divisible fleets obtained in 20 minutes of computational time

Table 5

\begin{tabular}{|l|c|c|c|c|c|c|c|c|c|c|}
\hline \multicolumn{1}{|c|}{ Instances: } & Bratislava & $\begin{array}{c}\text { Dubnica } \\
\text { nad } \\
\text { Vahom }\end{array}$ & Hradza & Kosice & $\begin{array}{c}\text { Liptovsky } \\
\text { Mikulas }\end{array}$ & Leopoldov & Michalovce & Nitra & $\begin{array}{c}\text { Nove } \\
\text { Zamky }\end{array}$ & Puchov \\
\hline No. of F. & 14 & 10 & 16 & 13 & 9 & 11 & 10 & 10 & 9 & 12 \\
\hline No. of EP. & 25 & 25 & 26 & 25 & 25 & 25 & 25 & 25 & 25 & 25 \\
\hline Rows & 2083 & 3449 & 5000 & 1508 & 3555 & 1135 & 4185 & 2357 & 4022 & 2323 \\
\hline Columns & 1457 & 1517 & 2564 & 1243 & 1412 & 965 & 1581 & 1339 & 1435 & 1543 \\
\hline Tmax [min] & 162 & 938 & 202 & 184 & 668 & 140 & 1420 & 423 & 1497 & 278 \\
\hline Tbest [min] & $\mathbf{8 1}$ & $\mathbf{3 6 8}$ & $\mathbf{8 1}$ & $\mathbf{9 2}$ & $\mathbf{2 6 2}$ & $\mathbf{9 3}$ & $\mathbf{4 8 6}$ & $\mathbf{1 6 8}$ & $\mathbf{6 3 7}$ & $\mathbf{1 3 9}$ \\
\hline
\end{tabular}

Results of numerical experiments for divisible fleets obtained in 40 minutes of computational time

Table 6

\begin{tabular}{|l|c|c|c|c|c|c|c|c|c|c|}
\hline \multicolumn{1}{|c|}{ Instances: } & Bratislava & $\begin{array}{c}\text { Dubnica } \\
\text { nad } \\
\text { Vahom }\end{array}$ & Hradza & Kosice & $\begin{array}{c}\text { Liptovsky } \\
\text { Mikulas }\end{array}$ & Leopoldov & Michalovce & Nitra & $\begin{array}{c}\text { Nove } \\
\text { Zamky }\end{array}$ & Puchov \\
\hline No. of F. & 14 & 10 & 16 & 13 & 9 & 11 & 10 & 10 & 9 & 12 \\
\hline No. of EP. & 25 & 25 & 26 & 25 & 25 & 25 & 25 & 25 & 25 & 25 \\
\hline Rows & 2083 & 3449 & 5000 & 1508 & 3555 & 1135 & 4185 & 2357 & 4022 & 2323 \\
\hline Columns & 1457 & 1517 & 2564 & 1243 & 1412 & 965 & 1581 & 1339 & 1435 & 1543 \\
\hline Tmax [min] & 162 & 938 & 202 & 184 & 668 & 140 & 1420 & 423 & 1497 & 278 \\
\hline Tbest [min] & $\mathbf{8 1}$ & $\mathbf{3 6 8}$ & $\mathbf{8 1}$ & $\mathbf{9 2}$ & $\mathbf{2 4 5}$ & $\mathbf{9 3}$ & $\mathbf{4 8 6}$ & $\mathbf{1 6 8}$ & $\mathbf{6 1 3}$ & $\mathbf{1 3 9}$ \\
\hline
\end{tabular}

To analyze the sensitivity of the best-found solution on the permitted computational time, we performed these numerical experiments once more for the time limit of 40 minutes. The results are shown in Table 6 and it can be easily found that they were improved only in two instances (Liptovsky Mikulas and Nove Zamky).

\section{Comparison and conclusions}

We suggested two approaches to the evacuation plan design problem. The first approach was based on the concept of indivisible fleets, which enables a direct formulation of the linear model and easy use of the IP-solver. Nevertheless, the linear model was too large and that is why we suggested a process of its shrinking by clustering evacuation vehicles into bigger fleets.

This process reduced the size of the associated model, but it brought an additional condition in the model and it caused that the resulting evacuation times turned worse. The overview in Table 7 shows that the best results were achieved for the biggest models. This result evoked an idea to formulate the evacuation problem for divisible fleets. This task was successfully solved by a complicated reformulation of the originally non-linear model to the linear one. Even if the new model is much more complicated than the first approach model, it has a much smaller size and enables to reach better results in the same computational time. This fact is demonstrated in Table 8, where the row "Divisible" reports on the best evacuation times obtained by the first approach and the row "Indivisible" reports on the times obtained by the second approach.

As we have the possibility to estimate the optimal solution using an iterative approach, we are able to compare the obtained results of both suggested approaches to the best-known results, which are reported in the row "Best Known" of Table 8. The row "Gap" of this table, where differences between the divisible fleet and the iterative approaches in percentage are given, shows that the suggested approaches constitute a promising way to the evacuation plan design.

An overview of the evacuation times achieved by the approach based on the indivisible fleet concept

Table 7

\begin{tabular}{|l|c|c|c|c|c|c|c|c|c|c|}
\hline \multicolumn{1}{|c|}{ Instances: } & Bratislava & $\begin{array}{c}\text { Dubnica } \\
\text { nad } \\
\text { Vahom }\end{array}$ & Hradza & Kosice & $\begin{array}{c}\text { Liptovsky } \\
\text { Mikulas }\end{array}$ & Leopoldov & Michalovce & Nitra & $\begin{array}{c}\text { Nove } \\
\text { Zamky }\end{array}$ & Puchov \\
\hline 1 vehicle & 81 & 434 & 93 & 92 & 294 & 101 & 586 & 182 & 704 & 139 \\
\hline 2 vehicles & 81 & 495 & 93 & 92 & 328 & 108 & 584 & 194 & 750 & 139 \\
\hline 4 vehicles & 81 & 510 & 93 & 92 & 381 & 105 & 796 & 198 & 1091 & 139 \\
\hline 8 vehicles & 81 & $*$ & 93 & 92 & $*$ & 119 & $*$ & 272 & $*$ & 139 \\
\hline Minimum & $\mathbf{8 1}$ & $\mathbf{4 3 4}$ & $\mathbf{9 3}$ & $\mathbf{9 2}$ & $\mathbf{2 9 4}$ & $\mathbf{1 0 1}$ & $\mathbf{5 8 6}$ & $\mathbf{1 8 2}$ & $\mathbf{7 0 4}$ & $\mathbf{1 3 9}$ \\
\hline
\end{tabular}


Table 7

\begin{tabular}{|l|c|c|c|c|c|c|c|c|c|c|}
\hline \multicolumn{1}{|c|}{ Instances: } & Bratislava & $\begin{array}{c}\text { Dubnica } \\
\text { nad } \\
\text { Vahom }\end{array}$ & Hradza & Kosice & $\begin{array}{c}\text { Liptovsky } \\
\text { Mikulas }\end{array}$ & Leopoldov & Michalovce & Nitra & $\begin{array}{c}\text { Nove } \\
\text { Zamky }\end{array}$ & Puchov \\
\hline Indivisible & 81 & 434 & 93 & 92 & 294 & 101 & 586 & 182 & 704 & 139 \\
\hline Divisible & $\mathbf{8 1}$ & $\mathbf{3 6 8}$ & $\mathbf{8 1}$ & $\mathbf{9 2}$ & $\mathbf{2 6 2}$ & $\mathbf{9 3}$ & $\mathbf{4 8 6}$ & $\mathbf{1 6 8}$ & $\mathbf{6 3 7}$ & $\mathbf{1 3 9}$ \\
\hline Best Known & 81 & 360 & 81 & 92 & 236 & 93 & 469 & 167 & 568 & 139 \\
\hline Gap [\%] & $\mathbf{0}$ & $\mathbf{2 . 2}$ & $\mathbf{0}$ & $\mathbf{0}$ & $\mathbf{1 1}$ & $\mathbf{0}$ & $\mathbf{3 . 6}$ & $\mathbf{0 . 6}$ & $\mathbf{1 2 . 1}$ & $\mathbf{0}$ \\
\hline
\end{tabular}

\section{References}

[1] JANACEK, J.: Service System Design in the Public and Private Sectors, Proc. of the international conference Quantitative Methods in Economics (Multiple Criteria Decision Making XII), 2004, Virt, ISBN 80-8078-012-9, pp. 101-108.

[2] JANACEK, J.: Time Accessibility and Public Service Systems, Proc. of conference Quantitative Methods in Economics, Bratislava, 2006, pp. 57-63.

[3] SIBILA, M.: Management and Transport of Inhabitant Evacuation (in Slovak), Dissertation thesis, Zilinská univerzita, Fakulta specialneho inzinierstva, Katedra technickych vied a informatiky, Zilina, 2009, p. 136.

[4] TEICHMANN, D.: Contribution to the Problems of Inhabitant Evacuation and Usage of Mathematical Programming for the Evacuation Planning (in Czech), Proc. Krizovy management, Vol. 7, 2/2008.

[5] XPRESS-MP Manual “Getting Started”. Dash Associates, Blisworth, UK, 2005, p. 105.

[6] XPRESS-Mosel “User guide”. Dash Associates, Blisworth, 2005, UK, p. 99.

Acknowledgment: This work was supported by project "Centre of excellence for systems and services of intelligent transport" ITMS 26220120028

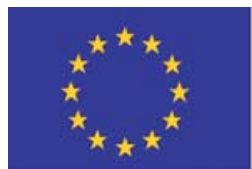

Project Part-Financed by the European Union

European Regional

Development Fund

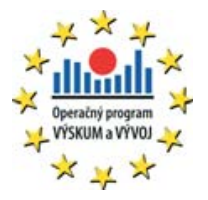

